\title{
BMJ Open Parents, healthcare professionals and other stakeholders' experiences of caring for babies born too soon in a low- resource setting: a qualitative study of essential newborn care for preterm infants in Kenya
}

\author{
Eleanor J Mitchell (10 , ${ }^{1}$ Phoebe Pallotti, ${ }^{2}$ Zahida P Qureshi, ${ }^{3}$ Jane P Daniels, \\ Mary Oliver, ${ }^{4}$ Fredrick Were, ${ }^{5}$ Alfred Osoti, ${ }^{3}$ George Gwako, ${ }^{3}$ Violet Kimani, ${ }^{6}$ \\ Jacqueline Opira, ${ }^{6}$ Shalini Ojha (1) ${ }^{7,8}$
}

To cite: Mitchell EJ, Pallotti P, Qureshi ZP, et al. Parents, healthcare professionals and other stakeholders' experiences of caring for babies born too soon in a low-resource setting: a qualitative study of essential newborn care for preterm infants in Kenya. BMJ Open 2021;11:e043802. doi:10.1136/ bmjopen-2020-043802

- Prepublication history and additional supplemental material for this paper are available online. To view these files, please visit the journal online (http://dx.doi.org/10.1136/ bmjopen-2020-043802).

Received 13 August 2020 Accepted 05 May 2021

D) Check for updates

(c) Author(s) (or their employer(s)) 2021. Re-use permitted under CC BY-NC. No commercial re-use. See rights and permissions. Published by BMJ.

For numbered affiliations see end of article.

\section{Correspondence to}

Eleanor J Mitchell;

Eleanor.Mitchell@nottingham. ac.uk

\section{ABSTRACT}

Objectives Prematurity is the leading cause of global neonatal and infant mortality. Many babies could survive by the provision of essential newborn care. This qualitative study was conducted in order to understand, from a family and professional perspective, the barriers and facilitators to essential newborn care. The study will inform the development of an early warning score for preterm and low birthweight infants in low and middle income countries (LMICs).

Setting Single-centre, tertiary referral hospital in Nairobi, Kenya.

Participants Nineteen mothers and family members participated in focus group discussions and 20 keyinformant interviews with professionals (healthcare professionals and policy-makers) were conducted. Focus group participants were identified via postnatal wards, the newborn unit and Kangaroo Mother Care (KMC) unit. Convenience and purposive sampling was used to identify professionals.

Outcome measures Understanding facilitators and barriers to provision of essential newborn care in preterm infants.

Results From 27 themes, three global themes emerged from the data: mothers' physical and psychological needs, system pillars and KMC.

Conclusion Meeting mothers' needs in the care of their babies is important to mothers, family members and professionals, and deserves greater attention. Functioning system pillars depended on a standardised approach to care and low cost, universally applicable interventions are needed to support the existing care structure. KMC was effective in both meeting mothers' needs, supporting existing care structures and also provided a space for the resolution of the dialectical relationship between families and hospital procedures. Lessons learnt from the implementation of KMC could be applied to the development of an early warning score in LMICs.
Strengths and limitations of this study

- This is the first report of parents', healthcare professionals' and wider stakeholders' views of essential newborn care in the context of development of an early warning score in low and middle income countries.

- A wide range of parents were recruited to ensure a wide range of opinions were elicited.

- Parents were recruited from a single, large national referral hospital, though some healthcare professionals had previous experience outside of this hospital.

\section{BACKGROUND}

Globally, 15 million infants are born prematurely (before 37 weeks gestation), the leading cause of neonatal mortality and morbidity. ${ }^{1}$ More than $60 \%$ of all preterm births occur in sub-Saharan Africa $^{2}$ and over a third of all neonatal deaths are due to preterm birth complications. ${ }^{3}$ In Kenya, the preterm birth rate is 8.6 per 1000 live births ${ }^{1}$ and neonatal mortality rate (NMR) is 20.9 deaths per 1000 live births, ${ }^{4}$ currently double the global goal of an NMR of $<10$ deaths per 1000 live births by $2035 .{ }^{3}$ In this setting, preterm infants should be managed in accordance with national and international evidence-based guidance for essential newborn care, ${ }^{56}$ including strategies for thermoregulation, resuscitation, feeding, Kangaroo Mother Care (KMC) and infection management. More than $80 \%$ of all preterm births occur between 32 weeks and 37 weeks gestation and most babies could survive with the provision of essential newborn care. ${ }^{2}$ 
This study is part of a wider programme of work developing an early warning score (EWS) for use in preterm and low birthweight (LBW) infants in low and middle income countries (LMICs). An EWS is used by healthcare professionals (HCPs) to document vital signs in a standardised way. While they are used for adults in a variety of clinical areas, ${ }^{7-9}$ there are few EWSs for newborn infants and none developed or used in LMICs. ${ }^{10}$ An EWS identifies vital signs that require closer monitoring and escalation of care which could result in a reduction in mortality and other serious morbidities. Before adopting an intervention developed for a high-resource setting in an LMIC, it is important to explore the current provision of care for the LMIC setting and to ensure the opinions of parents and HCPs are considered.

The aims of this study were to gain a contextual understanding of parents', HCPs' and other stakeholders' experiences of essential newborn care in Kenya, to understand barriers and facilitators to caring for a preterm or LBW infant in a low-resource setting and to consider both in the development of an EWS.

\section{METHODS}

This qualitative study was conducted at a large tertiary referral hospital in Nairobi (Kenyatta National Hospital $(\mathrm{KNH})$ ). Focus group discussions (FGDs) with mothers and family members were conducted in parallel to key informant interviews (KIIs) with a range of stakeholders involved in care of or management of preterm or LBW infants. FGDs were chosen for mothers and family members to reduce the risk of a potential power imbalance between participants and the researcher. A trusted staff member (clinical matron) was also invited to the FGDs to support the parents. Furthermore, the collaborative aspects of care were the focus of this study, involving both the relationships between the parents and the HCPs, but also the 'community' of mothers with sick babies. FGDs were the most appropriate methodology to capture this dynamic. KIIs were chosen for stakeholders for organisation and convenience reasons. The study was conducted with full ethical approval from the joint $\mathrm{KNH}-$ University of Nairobi Ethics Research Committee (reference number: P772/11/2018) and the Faculty of Medicine and Health Sciences Research Ethics Committee at the University of Nottingham (reference number: 161-1812) and is reported in accordance with the consolidated criteria for reporting qualitative research (COREQ). ${ }^{11}$

\section{Recruitment}

Training in interview techniques was provided to the team by PP, EJM and SO. Potential mothers and families were identified by a clinical matron on postnatal wards, the newborn unit and KMC unit. Mothers of different ages, different duration of hospital stay and singleton and multiple pregnancies were invited to ensure a diverse sample. Participant information sheets were provided in English or Kiswahili. Convenience and purposive sampling was used to recruit HCPs via the labour suite, postnatal wards and newborn unit. External stakeholders, including policy-makers and non-government organisation (NGO) representatives, were purposively sampled and invited to participate in an interview. Participants' involvement was somewhat determined by their availability, though the research team made efforts to accommodate an interview at a mutually convenient time. All participants gave their written informed consent prior to taking part.

\section{Data collection}

All data collection was undertaken by VK, a Kenyan female social scientist educated to PhD level with over 40 years' experience, during March and April 2019. Two FGDs, each lasting around 90 minutes, were conducted at KNH. The interview guide consisted of prompts to explore mothers' and family members' views of essential newborn care for a premature baby (online supplemental material 1). Face-to-face KIIs lasted around $60 \mathrm{~min}$. All discussions were audio-recorded and transcribed. No identifiable data were included on the transcripts.

\section{Analysis}

Thematic network analysis (TNA) was used. ${ }^{12}$ TNA is a way of coding, organising and identifying emergent themes from the data in a systematic way and improves the internal validity of the results by employing systematic and repeatable methods. Global themes emerged from the grouping of organising themes into more abstracted and/or structural categories and was done by both analysts in collaboration. The philosophical underpinnings of this study are relational dialectic theory ${ }^{13}$ as a pragmatic but nuanced way of identifying effective clinical interventions, which would work within the human context of neonatal care in a less resourced setting where much of the burden of basic nursing care rests on the mothers. Understanding what is fairly pragmatic data through this epistemological lens allowed the researchers to elucidate the spaces where communication and relationship between mothers and HCPs are either enabled or prevented. This understanding also removes the concept of 'blame' of failures in care from either side, preferring a more salutogenic approach to designing protocols and practices which support collaborative working. ${ }^{14}$ Data from FGDs and KIIs were analysed together in order to consider consistent themes and gaps between the two. Table 1 shows an example of a coding tree. Initial coding was undertaken independently by EJM and PP before being moderated by a third reviewer (MO). Analyses were conducted using NVivo V.12 (QSR international). Initial codes were organised into organising and global themes in accordance with the stages of TNA.

The study is strengthened by the fact that data collection and analyses were performed by teams in Kenya and the UK, since concepts are developed from a more etic perspective. However, although one of the senior academic analysts has substantial clinical experience in 


\begin{tabular}{|c|c|c|}
\hline Global theme & $\begin{array}{l}\text { Organising } \\
\text { theme }\end{array}$ & Basic semantic code \\
\hline $\begin{array}{l}\text { Mother's } \\
\text { physical and } \\
\text { psychological } \\
\text { needs }\end{array}$ & $\begin{array}{l}\text { Impact } \\
\text { on wider } \\
\text { family and } \\
\text { community }\end{array}$ & $\begin{array}{l}\text { Impact of children on mother not } \\
\text { being at home } \\
\text { Long inpatient stay } \\
\text { Involvement of other siblings } \\
\text { Emotional impact of preterm } \\
\text { death on siblings } \\
\text { Impact on siblings has led to drop } \\
\text { in educational performance } \\
\text { Anxiety to mothers around leaving } \\
\text { older siblings at home } \\
\text { Impact on other children and } \\
\text { family } \\
\text { Preterm birth impacts on other } \\
\text { children } \\
\text { Daddy panics about mum's health } \\
\text { Involvement of dads and wider } \\
\text { family } \\
\text { Less involvement from daddy } \\
\text { Partner anxiety about premature } \\
\text { baby } \\
\text { 'You are not a burden' family } \\
\text { member perspective }\end{array}$ \\
\hline
\end{tabular}

a similar setting, the lack of lived experience in the exact context means that more nuanced cultural understandings may well have been missed as a result. However, all results were discussed with the wider team (Kenya and the UK). The positionality of the researchers was discussed at each stage of data collection and analysis. Thought was given particularly to the potential influence of the interviewer in terms of their familiarity with some participants (HCPs) and potential deference of either the researcher or participants, depending on the relationship therein.

\section{Role of funding source}

The funder had no role in the collection, analysis or interpretation of data, writing of the report or decision to submit the paper for publication.

\section{Patient and public involvement}

Patients and the public were not involved in the design or conduct of this study. However, the views of parents and stakeholders involved in this study will help to shape future research in this area.

\section{RESULTS}

\section{Participants}

Focus groups: 19 mothers were invited and all agreed to participate in the FGD; three of which were accompanied by the infant's father and two by other family members (mother-in-law and sister). All mothers had experience of their infant being on the newborn unit and 10 also had experience of the postnatal ward. Infants of the participants were born between 27 weeks and 34 weeks gestation and, on average had spent 20 days in hospital since birth. Participants' characteristics are shown in table 2.

\begin{tabular}{|c|c|c|c|}
\hline $\begin{array}{l}\text { Focus } \\
\text { group }\end{array}$ & $\begin{array}{l}\text { Gestational } \\
\text { age (weeks) of } \\
\text { infant }\end{array}$ & $\begin{array}{l}\text { Experience } \\
\text { of post-natal } \\
\text { ward }\end{array}$ & $\begin{array}{l}\text { Infant's length } \\
\text { of stay (days) } \\
\text { at time of } \\
\text { focus group } \\
\text { discussion }\end{array}$ \\
\hline \multirow[t]{9}{*}{1} & 34 & No & 18 \\
\hline & 29 & Yes & 26 \\
\hline & 34 & No & 12 \\
\hline & 32 & No & 30 \\
\hline & 32 & Yes & 30 \\
\hline & 32 & Yes & 19 \\
\hline & 32 & No & 17 \\
\hline & 33 & No & 9 \\
\hline & 29 & Yes & 23 \\
\hline \multirow[t]{10}{*}{2} & 27 & Yes & 10 \\
\hline & 32 & No & 47 \\
\hline & 30 & No & 7 \\
\hline & 31 & Yes & 33 \\
\hline & 31 & No & 16 \\
\hline & 31 & Yes & 44 \\
\hline & 32 & No & 14 \\
\hline & 33 & Yes & 10 \\
\hline & 28 & Yes & 10 \\
\hline & 34 & Yes & 13 \\
\hline
\end{tabular}

KIIs: 31 stakeholders were invited to participate and 20 KIIs were conducted. Stakeholder characteristics are given in table 3.

\section{THEMES}

In total, 153 basic semantic codes emerged from the data, which were then synthesised into 27 organising codes. Three global themes emerged from the organising codes: (1) mothers' physical and psychological needs, (2) system pillars and (3) KMC. The organising codes for each global theme are shown in figures 1-3.

The three themes were emergent from the process of analysing the organising themes into overarching categories, which were either more abstracted in terms of concepts (for instance, the wider issue of mothers' biopsychosocial needs) or structures of care. An example of how the third global theme, $\mathrm{KMC}$, emerged is given in table 4 .

\section{Global theme 1: mothers' physical and psychological needs}

This theme reflected the met and unmet needs of mothers, families and the wider community, while the baby was in hospital.

Mothers and fathers were generally positive about the support and communication from staff and their own communities, though there was also a recurrent theme of basic physical needs being unmet. This included a lack 


\begin{tabular}{lll}
\hline Table 3 & KIls participant characteristics & \\
\hline $\begin{array}{l}\text { Participant } \\
\text { number }\end{array}$ & Stakeholder type & $\begin{array}{l}\text { Years of } \\
\text { professional } \\
\text { experience }\end{array}$ \\
\hline 1 & Policy-maker & 9 \\
\hline 2 & NGO representative & 28 \\
3 & Obstetrician & 43 \\
\hline 4 & Paediatrician & 50 \\
\hline 5 & Paediatrician & 35 \\
\hline 6 & Obstetrician & 12 \\
\hline 7 & NGO representative & 13 \\
\hline 8 & Obstetrician & 15 \\
\hline 9 & Nurse & 7 \\
\hline 10 & Nurse & 23 \\
\hline 11 & Neonatal Nurse & 20 \\
\hline 12 & Neonatal Nurse & 27 \\
\hline 13 & Neonatal Nurse & 4 \\
\hline 14 & Nurse & 20 \\
\hline 15 & Neonatal nurse & 20 \\
\hline 16 & Neonatal nurse & 9 \\
\hline 17 & Nurse & 4 \\
\hline 18 & Nurse & 3 \\
\hline 19 & Nurse & 25 \\
\hline 20 & Nurse & 14 \\
\hline & & \\
\hline
\end{tabular}

KIls, key informant interviews; NGO, non-government organisation.

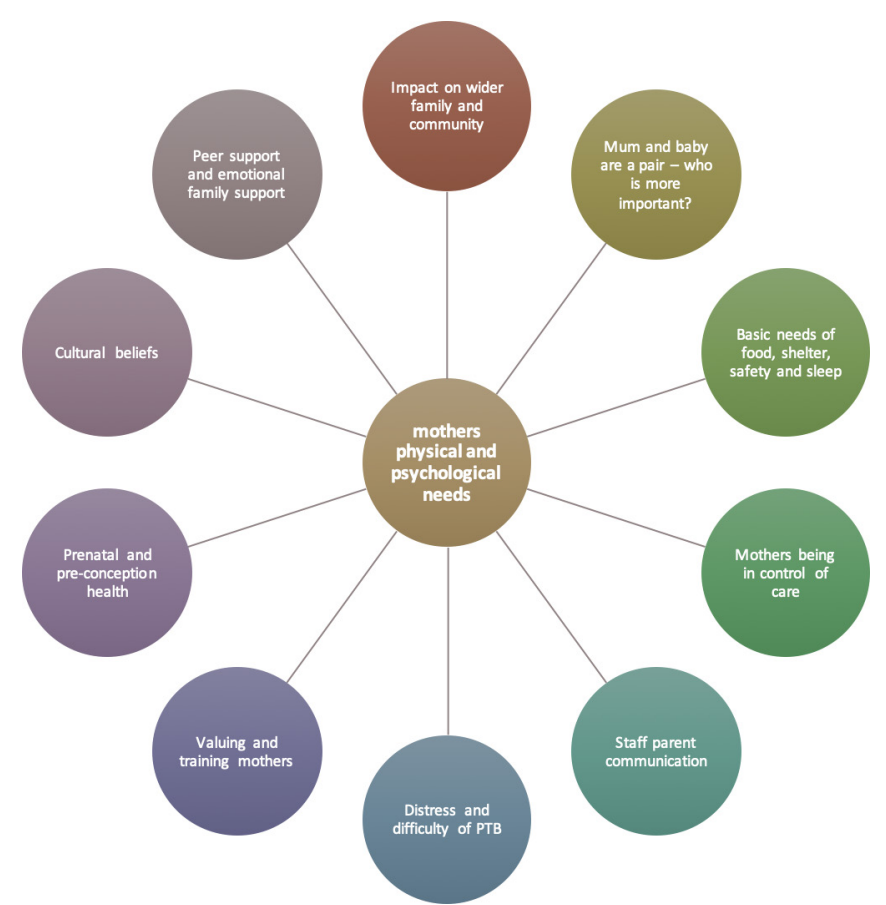

Figure 1 Global theme 1: mothers' physical and psychological needs.

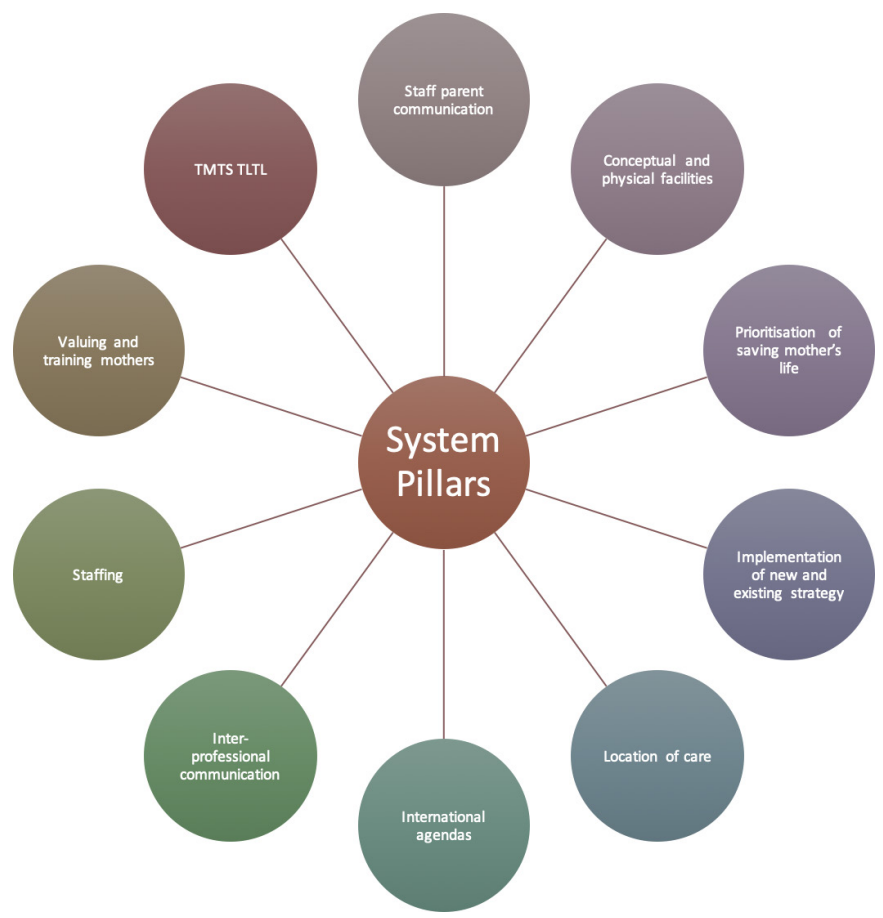

Figure 2 Global theme 2: system pillars.

of available food and drink, feeling unsafe while moving around the hospital at night and a scarcity of places to sleep.

'the mother must feed well in order to produce milk. Like the mothers here, we have a schedule to feed the babies and then do kangaroo care for about an hour. Every two hours the routine is repeated. Most of the time the schedules coincide with meal times ... . You go back to the ward and there's no food!' (Mother, FGD 2)

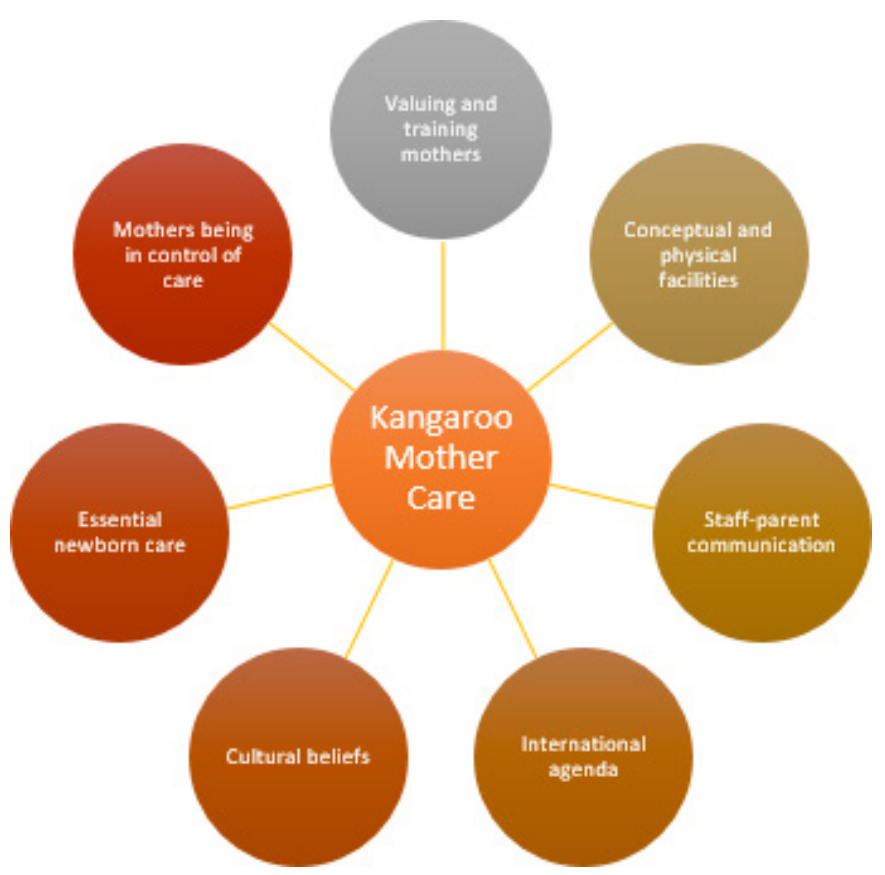

Figure 3 Global theme 3: Kangaroo Mother Care. 
Table 4 An example of how the third global theme, KMC, emerged

\begin{tabular}{ll} 
Basic codes & Organising themes \\
\hline Kangaroo care helps mum cope & Mothers being in control of care \\
KMC as a simple intervention & Valuing and training mothers \\
Low-tech solutions to lack of high-tech solutions & Conceptual and physical facilities \\
Simple interventions & Staff parent communication \\
KMC can be seen as a primitive practice & International agenda \\
Encouragement that KMC helps baby grow & Cultural beliefs \\
High-level strategy helped develop KMC & Essential newborn care \\
(including generational effects) & Impact on wider family and community \\
KMC=mother and baby bond & \\
KMC allows mum to always be with baby & \\
KMC keeps your baby warm & \\
KMC and feeding & \\
Better brains - ongoing development & \\
Importance of baby staying with mum & \\
KMC improves weight gain & \\
Role of KMC in infection reduction &
\end{tabular}

KMC, Kangaroo Mother Care.

Impact of extended inpatient stays on the wider families were noticeably absent from the KIIs but referred to frequently in the FGD data.

'My first child is twelve-year-old and is at home. I delivered preterm twins about a month ago. One died on the third day and I was called to see the body before they removed him. My child was informed I delivered twins but he did not get to see them. Children are not allowed in this unit. He keeps wondering when I will go back home. I worry a lot about my child.' (Mother, FGD 2)

One of the most positive themes was the value placed on the mothers' care (and in some cases, other family members) of the baby. The newborn unit is crowded and the staff to patient ratio is low, so training and valuing the mothers input is a pragmatic solution, but the concept of maternal love and the importance of the mother baby bond also added an extra emotional and spiritual dimension to this theme.

'We feel included in everything to do with our baby care. We alert the nurse if baby seems uncomfortable. You get used to your baby. And if you have any question, you ask nurse or matron.' (Mother, FGD 1)

The impact on and support of the wider community was seen as key. The mother's absence from the home and the community costs (direct and indirect) was noted but families and staff also spoke of the importance of support and training from their communities.

'We intervene at grassroots level. We provide guidelines and capacity building through training at community level.' (Participant 2, KII)

Cultural aspects of the conceptualisation of preterm birth led some mothers to feel stigmatised, but where community and wider support was given, families felt the outcomes were better for all.

'When I delivered this baby even my husband was anxious, let me say disappointed. He stated, 'why did you deliver this one?' This is 'Mtoto wa kisirani.' ... (meaning an ill-fated baby).' (Mother, FGD 1)

\section{Global theme 2: system pillars}

This theme refers to the intra-hospital and pan-Kenyan organisation of care for premature and LBW babies. Much of this data is taken from the KIIs though mothers and families did comment on the structures of care within their units.

Using the "Too Much Too Soon, Too Little Too Late (TLTL)' model, ${ }^{15}$ we initially examined the data on resources allocated to the care of premature babies. Overall, the Kenyan context seemed to indicate that the latter (TLTL) was the most problematic in terms of both the incidence of preterm birth and the survival rate thereafter. Stakeholders discussed resource issues at length, particularly in regards to differences between rural and urban (and tertiary and primary) care settings.

'If we have to reduce infant deaths we must ensure mothers are aware of danger signs. This can be addressed during ANC clinics. For example, if a mother notices spotting they should go for check-up.'

(Participant 7, KII)

Facilities were mentioned by almost all stakeholders, irrespective of role or years of experience. The crucial issue in preterm care seemed to be physical space, with nurseries being overcrowded and thus adequate monitoring and infection control being almost impossible to reliably achieve. 
'I mean in the cots in unit X. Some babies have long legs, so they have to be folded in order to share the limited space with others. The baby cannot sleep freely as they are squeezed.' (Mother, FGD 2)

Stakeholders discussed recent technological and care protocol advances in neonatal care and the adoption across Kenya. Newer equipment and medicines seemed to be mostly available in the urban units, but adaptations in rural areas were also discussed.

'I would like this simple technology to continue, particularly in facilities that are not well equipped. Then put a sufuria [cooking pot] of boiling water at a corner of the room. The humidity acts like an incubator.' (Participant 3, KII)

More strategic stakeholders discussed that national and international training programmes were constantly rolled out, and while staff retention was a problem, upskilling of existing staff was felt to be on an upward trajectory.

'We developed a curriculum for training for use. Right now our staff is in [a region] developing training curriculum. We have trained over 9000 health care staff in the past three to four years.' (Participant $1, \mathrm{KII})$

Finally, the implementation of new and existing protocols and the roll-out of new technology and training, in line with key global priorities, was discussed by all stakeholders.

'As we evolved now we have ventilators and at [the hospital] we have even mechanical ventilators. We also have CPAP [continuous positive airway pressure] machines.' (Participant 4, KII)

\section{Global theme 3: KMC}

KMC units were identified as a valued and useful intervention in the FGDs and the KIIs alike.

For mothers and families, being an integral part of the infant's care on a KMC unit was seen as psychologically supportive. They felt more positive about the baby's chances of survival, more comfortable balancing their own needs with the needs of their feeding baby and more involved in their wider care.

'When a baby is weighed today, there is an increase from yesterday's weight and tomorrow weight will be higher than today. I attribute this to kangaroo care. The mother is always with her baby. The baby grows fast.' (Mother, FGD 1)

Furthermore, 'baby wearing' was seen as culturally acceptable and considered a normal way to care for any infant, normalising the practice and easing the transition from hospital to home.

'In our traditional setting, we don't like pushing our babies as they look in empty space [a pram]. We like direct contact with our babies. In this setting then
Kangaroo mother care is commendable and embraced much more easily.' (Participant 3, KII)

Participants also referred to the benefits of KMC in alleviating infrastructure and resource issues and the wider Government strategy for roll-out of KMC.

'You can look at it from the infrastructure. Incubators are obviously inadequate for the number of babies needing them ..., but KMC is skin to skin and need no regulation.' (Participant 7, KII)

\section{DISCUSSION}

This study gives voice to mothers and families, HCPs, and wider stakeholders in Kenya. Its results empower them to be heard and understood such that any further plans to improve the care for preterm and LBW infants in this setting are built on their priorities. The emergent themes are discussed here in the context of the development of an EWS for preterm infants in LMICs, with the third global theme, 'KMC', considered an exemplar of an intervention which meets the needs' of the two previous global themes. Public partnership and stakeholder engagement in research and quality improvement initiatives are of particular importance in low resource settings, since it encourages participation from previously marginalised populations. ${ }^{16}$ The results of this study were shared with a group of 78 stakeholders with a key interest in preterm birth.

Participants in the KIIs discussed the importance of the development and implementation of simple interventions and initiatives supported by the government, ensuring that such initiatives were aligned with national and global priorities for newborn health. If an EWS was developed for use in an LMIC, it would, therefore, be important to ensure 'buy-in' from a wide range of stakeholders, including the state. Consistent and standardised approaches to the provision of healthcare for newborn infants was considered important. Any effective interventions should be scaled up rapidly to all groups within countries, in line with the WHO's goal to provide universal health coverage and to enable progress towards the Sustainable Development Goals by $2030 .{ }^{17}$ KII participants commented on lack of infant monitoring and poor documentation on the newborn unit. This is consistent with an observational study conducted by the same team showing record-keeping was poor with many infants' having few vital signs recorded ${ }^{18}$ and a previous newborn study in Nairobi showing that vital signs charts were only completed for around a third of infants. ${ }^{19}$ These issues are not uncommon in low-resource settings. A qualitative study of health facility leaders in Ethiopia also found that human resource issues such as training and staffing levels are critical in caring properly for preterm infants. ${ }^{20}$

Mothers, other family members and HCPs all placed importance on the mother's involvement in their newborn infant's care and, in particular, the communication 
between the HCP and the mother. The EWS provides a standardised method of monitoring infants, which could also provide more focused communication between the HCP and mother. It could serve as a visual aid to the mother, so she can easily see how her baby is doing; however, this would need to be done with care and sensitivity to avoid causing undue anxiety. Communication with parents would be an essential element to be included in the provision of training provided to HCPs.

The third global theme that emerged was KMC, an evidence-based intervention commonly practiced in this setting, which was considered an exemplar where the resources and issues of each group, and the tensions between them, are met in and answered by the KMC unit. From a staff and system perspective, KMC units alleviated some of the resource challenges in the Kenyan context. Fewer incubators and fewer interventions with feeding and infection control were necessary and participants felt that babies were usually discharged home earlier. Importantly, KMC is strongly supported by the government who are working with NGOs to roll-out this initiative more widely across the country. From a strategy perspective, $\mathrm{KMC}$ is an established priority and included in national guidance. ${ }^{21}$ The emergence of KMC as a strong theme demonstrated that any new intervention developed must be suitably adopted to support uninterrupted KMC.

The key principles of relational dialectics, contradictions, totality, process and praxis, whereby seemingly opposing needs, views and ontological perspectives, are processed and brought to agreement and then practically implemented ${ }^{13}$ are met in this study. If, as the mothers do so much of the 'work' of nursing a sick baby, one sees them as colleagues as well as patients of the HCPs, understanding the blending of roles and relationships inherent in this may well open spaces of both better communication and a better mutual meeting of needs and responsibilities. The principal exemplar of this is in the provision of KMC units, which served both mothers' physical and psychological needs, reduced the burden of care and interventionist medicine on the healthcare system both on a unit and hospital level and allowed more discussion and co-production of care between mothers and HCPs on the unit. In this way, the physical existence of the KMC unit, as opposed to the practice of KMC itself, becomes vital for enabling this relational collaboration to exist in the 'openness' it engenders. ${ }^{14}$ The enthusiasm and praise for the $\mathrm{KMC}$ unit is seen clearly in the data from all stakeholders and this can be seen as a practical and as an emotive value placed on that, which makes the participants feel at ease and feel successful in their shared goals of the babies' care. ${ }^{13}$

KMC units are highly valued, from a mother's perspective, ensuring use, ${ }^{22}$ they meet national and international guidelines for the care of premature and LBW babies $^{23}$ and they use existing resources-beds, nurses and mothers. ${ }^{24}$ However, roll-out of facility-based KMC, at scale, is not without challenges. A systematic review of implementation of KMC from a health systems perspective
(86 studies) identified a range of barriers and enablers, including buy-in from staff, availability of training and resources and time to train and provide KMC. ${ }^{25}$ Research focusing on the implementation of KMC is indeed in the top 10 research priorities for improving newborn health and birth outcomes by $2025 .{ }^{26}$ This is in contrast to the possible difficulties in implementing an intervention on newborn units, which relies heavily on having adequate resources in place and, in particular, the availability of senior clinicians to see acutely unwell infants and expensive technology to support thermoregulation, infection control and infant feeding. This issue was discussed in our meeting of 78 stakeholders in Kenya, all of whom are involved in the care or provision of care for newborn infants. While the majority of stakeholders were positive about the concept of EWS, human resource issues were seen as a key barrier that would need to be overcome for successful implementation. ${ }^{18}$ In addition, there is a strong evidence base for KMC. Studies have shown it improves outcomes for neonates, especially preterm and LBW babies, ${ }^{27} 28$ which in turn reduces the financial and strategic burden on paediatric services as a whole in this context. Therefore, mothers, HCPs and policymakers can clearly see value and benefit in its provision and implementation. For an EWS to be implemented widely across LMICs, in addition to the potential benefits of standardised record-keeping and visual simplicity of such a chart, evidence would need to be generated in its ability to reduce neonatal mortality and morbidities. Further work has been funded to examine the feasibility and acceptability of implementing the EWS on several newborn units in Kenya and the key themes drawn from this study will ensure that the next step of this programme of work considers the views of mothers, families, HCPs and other stakeholders. If feasibility is demonstrated, then a study to investigate the possible clinical benefits would be warranted.

This is the first study to report views on the provision of newborn care in the context of the development of an EWS for preterm and LBW infants in LMICs. A limitation of the study is that mothers and families were recruited from a single national tertiary referral hospital and, therefore, may not represent wider views. However, many HCPs who were interviewed, while based at $\mathrm{KNH}$, also had clinical experience outside of this single hospital, in addition to the wider expertise of two representatives at a national level.

In summary, while, at least theoretically, an EWS could potentially reduce neonatal mortality and serious morbidities, this is, as yet, unknown. If these potential benefits of an EWS were to be demonstrated in future studies, many of the issues identified in this study, including lack of space and overcrowding on newborn units, lack of trained neonatal staff and family involvement and communication, could be met. Results from this study may also help to identify areas for improvement in the provision of essential newborn care in a low-resource setting. For example, developing strategies to improve 
communication between mothers and HCPs and ways in which interventions, shown to be effective, can be rolled out rapidly across different levels of healthcare.

\section{CONCLUSIONS}

Ensuring the needs of parents, HCPs and policy-makers in the development of any intervention is important. A range of issues emerged that will be important to consider in the development and potential implementation of an EWS and lessons can be learnt from the emergent theme of KMC. KMC is already widely accepted as a gold standard intervention to increase newborn survival, especially in the preterm and LBW populations. ${ }^{28}$ This paper adds the valuable perspective that KMC units may also help to alleviate system pressures, both within units and in a national health provision and is invaluable to mothers and families in terms of their physical and psychological well-being.

\section{RESEARCH IN CONTEXT}

\section{Evidence before this study}

In the context of the development of an EWS, we conducted a literature search using PubMed in June 2018 for studies on neonatal EWSs, using the search terms 'early warning score' and 'neonatal early warning score'. We identified only a small handful of publications relating to neonatal EWS and one non-systematic review of available EWS; however, none were from LMICs. We found no studies which included the views of parents in the development of an EWS.

\section{Added value of this study}

This is the first study to importantly include the views and opinions of parents, family members, HCPs, policy-makers and non-government representatives in the context of the provision of newborn care and development of an EWS. A range of issues, important to parents/family members and stakeholders should be considered if an EWS were to be implemented, including: ensuring a consistent and standardised approach to newborn care, communication between parents and HCPs, and parental involvement in the newborn's care. KMC was viewed positively by all participants and should be considered as an exemplar in the development of other initiatives to improve newborn care.

\section{Implications of all the available evidence}

The perspectives of a wide range of stakeholders, including parents and wider family members, should be considered in the development of an intervention designed to improved newborn care. In the context of an LMIC setting, where KMC is a commonplace, any new intervention implemented should be considered alongside KMC. Further research is required to test the feasibility and acceptability of an EWS in hospitals in LMICs.
Author affiliations

${ }^{1}$ Nottingham Clinical Trials Unit, University of Nottingham, Nottingham, UK ${ }^{2}$ Maternal Health and Wellbeing Research Group, School of Health Sciences, University of Nottingham, Nottingham, UK

${ }^{3}$ Department of Obstetrics and Gynaecology, University of Nairobi, Nairobi, Kenya ${ }^{4}$ School of Education, University of Nottingham, Nottingham, UK

${ }^{5}$ Department of Paediatrics and Child Health, University of Nairobi, Nairobi, Kenya

${ }^{6}$ Kenya Paediatric Research Consortium, Nairobi, Kenya

${ }^{7}$ Population and Applied Health Sciences, School of Medicine, University of Nottingham, Nottingham, UK

${ }^{8}$ Neonatal Unit, University Hospitals of Derby and Burton NHS Foundation Trust, Derby, UK

Twitter Eleanor J Mitchell @eleanor_mitch

Acknowledgements We thank all participants and our funders.

Contributors EJM was the principal investigator; conceived the idea, analysed data and wrote the first draft of the manuscript. PP analysed data and had a major role in writing the manuscript. SO was the clinical lead for the study. MO moderated analysed data. ZPQ was the lead at the Kenyatta National Hospital (KNH), supported by J0 who provided study coordination. Qualitative data collection was undertaken by VK. FW, AO and GG all provided clinical input at KNH. JD provided mentorship to the team. All authors contributed to interpretation of the data, draft manuscripts and approved the final version of the manuscript prior to submission.

Funding University of Nottingham Global Challenges Research Fund (GCRF) (Ref: RIS 630122).

Competing interests SO declares grants from NIHR. No other conflicts of interest are declared by the authors.

Patient consent for publication Not required.

Ethics approval Ethical approval was granted by the joint Kenyatta National Hospital-University of Nairobi Ethics Research Committee (reference number: P772/11/2018) and the Faculty of Medicine and Health Sciences' Research Ethics Committee at the University of Nottingham (reference number: 161-1812).

Provenance and peer review Not commissioned; externally peer reviewed.

Data availability statement Data are available upon reasonable request to the corresponding author.

Supplemental material This content has been supplied by the author(s). It has not been vetted by BMJ Publishing Group Limited (BMJ) and may not have been peer-reviewed. Any opinions or recommendations discussed are solely those of the author(s) and are not endorsed by BMJ. BMJ disclaims all liability and responsibility arising from any reliance placed on the content. Where the content includes any translated material, BMJ does not warrant the accuracy and reliability of the translations (including but not limited to local regulations, clinical guidelines, terminology, drug names and drug dosages), and is not responsible for any error and/or omissions arising from translation and adaptation or otherwise.

Open access This is an open access article distributed in accordance with the Creative Commons Attribution Non Commercial (CC BY-NC 4.0) license, which permits others to distribute, remix, adapt, build upon this work non-commercially, and license their derivative works on different terms, provided the original work is properly cited, appropriate credit is given, any changes made indicated, and the use is non-commercial. See: http://creativecommons.org/licenses/by-nc/4.0/.

ORCID iDs

Eleanor J Mitchell http://orcid.org/0000-0002-6998-4533

Shalini 0jha http://orcid.org/0000-0001-5668-4227

\section{REFERENCES}

1 Chawanpaiboon S, Vogel JP, Moller A-B, et al. Global, regional, and national estimates of levels of preterm birth in 2014: a systematic review and modelling analysis. Lancet Glob Health 2019;7:e37-46.

2 World Health Organisation. Born too soon: the global action report on preterm birth; 2012.

3 World Health Organisation. Every newborn: an action plan to end preventable deaths; 2014

4 Unicef child mortality estimates: regional and global neonatal mortality rate. Available: http://data.unicef.org [Accessed 25 July 2019]. 
5 Republic of Kenya Ministry of Health. Basic paediatric protocol. January; 2016

6 World Health Organisation. Pregnancy, childbirth, postpartum and newborn care: a guide for essential practice; 2015 .

7 Morgan R L-WF. An early warning scoring system for detecting developing critical illness. Clin Intensive Care 1997;8.

8 Goldhill DR, McNarry AF. Physiological abnormalities in early warning scores are related to mortality in adult inpatients. $\mathrm{Br} J$ Anaesth 2004;92:882-4.

$9 \mathrm{Gao} \mathrm{H}, \mathrm{McD}$ onnell A, Harrison DA, et al. Systematic review and evaluation of physiological track and trigger warning systems for identifying at-risk patients on the ward. Intensive Care Med 2007;33:667-79.

10 Mortensen N, Augustsson JH, Ulriksen J, et al. Early warning- and track and trigger systems for newborn infants: a review. J Child Health Care 2017;21:112-20.

11 Tong A, Sainsbury P, Craig J. Consolidated criteria for reporting qualitative research (COREQ): a 32-item checklist for interviews and focus groups. Int J Qual Health Care 2007;19:349-57.

12 Attride-Stirling J. Thematic networks: an analytic tool for qualitative research. Qualitative Research 2001;1:385-405.

13 Baxter LA. A dialectical perspective of communication strategies in relationship development. Handbook of Personal Relationships: Wiley, 1988.

14 Baxter LA, Bridge K. Blended relationships: friends as work associates. Western journal of communication 1992;56:200-25

15 Miller S, Abalos E, Chamillard M, et al. Beyond too little, too late and too much, too soon: a pathway towards evidence-based, respectful maternity care worldwide. Lancet 2016;388:2176-92.

16 Cohen ERM, Masum H, Berndtson K, et al. Public engagement on global health challenges. BMC Public Health 2008;8:168.

17 Boerma T, Requejo J, Victora CG, et al. Countdown to 2030: tracking progress towards universal coverage for reproductive, maternal, newborn, and child health. Lancet 2018;391:1538-48.
18 Mitchell EJ, Qureshi ZP, Were F, et al. Feasibility of using an early warning score for preterm or low birthweight infants in a lowresource setting: results of a mixed-methods study at a national referral hospital in Kenya. BMJ Open 2020;10:e039061.

19 Murphy GAV, Gathara D, Mwachiro J, et al. Effective coverage of essential inpatient care for small and sick newborns in a high mortality urban setting: a cross-sectional study in Nairobi City County, Kenya. BMC Med 2018;16:72.

20 Usman AK, Wolka E, Tadesse Y, et al. Health system readiness to support facilities for care of preterm, low birth weight, and sick newborns in Ethiopia: a qualitative assessment. BMC Health Serv Res 2019;19:860.

21 Repulic of Kenya Ministry of Health. Kangaroo mother care clinical implementation guidelines; 2016.

22 World Health Organisation Department of Reproductive Health and Research. Kangaroo mother care: a practical guide; 2003.

23 World Health Organisation. Recommendations on interventions to improve preterm birth outcomes; 2015.

24 Campbell-Yeo ML, Disher TC, Benoit BL, et al. Understanding kangaroo care and its benefits to preterm infants. Pediatric Health Med Ther 2015;6:15-32.

25 Chan G, Bergelson I, Smith ER, et al. Barriers and enablers of kangaroo mother care implementation from a health systems perspective: a systematic review. Health Policy Plan 2017;32:1466-75.

26 Yoshida S, Martines J, Lawn JE, et al. Setting research priorities to improve global newborn health and prevent stillbirths by 2025. J Glob Health 2016;6:010508.

27 Boundy EO, Dastjerdi R, Spiegelman D, et al. Kangaroo mother care and neonatal outcomes: a meta-analysis. Pediatrics 2016;137:e20152238.

28 Conde-Agudelo A, Díaz-Rossello JL. Kangaroo mother care to reduce morbidity and mortality in low birthweight infants. Cochrane Database Syst Rev 2016;8:CD002771. 